\title{
Spectral analysis of the Forel-Ule ocean colour comparator scale
}

\author{
M. R. Wernand \\ marcel.wernand@nioz.nl
}

\section{H. J. van der Woerd}

Royal Netherlands Institute for Sea Research, Physical Oceanography, Marine Optics \& Remote Sensing, PO box 59, 1790AB Den Burg, Texel, The Netherlands

Institute for Environmental Studies (IVM), VU University Amsterdam, De Boelelaan 1087, 1081 HV Amsterdam, The Netherlands

François Alphonse Forel (1890) and Willi Ule (1892) composed a colour comparator scale, with tints varying from indigo-blue to cola brown, to quantify the colour of natural waters, like seas, lakes and rivers. For each measurement, the observer compares the colour of the water above a submersed white disc (Secchi disc) with a hand-held scale of pre-defined colours. The scale can be well reproduced from a simple recipe for twenty-one coloured chemical solutions and because the ease of its use, the Forel-Ule (FU) scale has been applied globally and intensively by oceanographers and limnologists from the year 1890. Indeed, the archived FU data belong to the oldest oceanographic data sets and do contain information on the changes in geobiophysical properties of natural waters during the last century. In this article, we describe the optical properties of the FU scale and its ability to cover the colours of natural waters, as observed by the human eye. The recipe of the scale and its reproduction is described. The spectral transmission of the tubes and their respective chromaticity coordinates are presented. The FU scale, in all its simplicity, is found to be an adequate ocean colour comparator scale. The scale is well characterized, stable and observations are reproducible. Thus, the large historic data sets of FU measurements are coherent and well calibrated. Moreover, the scale can be coupled to contemporary multi-spectral observations with hand-held and satellite-based spectrometers. A reintroduction of the FU scale is recommended to expand the historical database and to facilitate a tie-in with present satellite ocean colour observations by tranforming MERIS normalized multi-band reflectance image into a FU indexed image. [D0I: 10.2971/je0s.2010.10014S]

Keywords: Forel-Ule scale, reflectance, transmission, ocean colour, chromaticity

\section{INTRODUCTION}

Colour classification of natural waters started at the end of the 19th century with the introduction of the Forel-Ule scale (FU scale). In 1890, François Alphonse Forel proposed his colour standard, a type of a combination of a cyanometer and xanthometer [1], to classify the blue to green waters [2]. A paper version of this scale is shown in Figure 1. Initially, the tube colours were given as a percentage of the Yellow agent (Potassium-chromate) added to the basic Blue (Copper sulphate) solution (percentage below the coloured bars of Figure 1). In 1892, Willi Ule [3] complemented the scale by adding the blue-green to brown colours. The combined scale (Forel's 1 to 11 and Ule's 12 to 21) became known as the Forel-Ule scale.

The mathematician Von Drygalski and the German geographer Krümmel (located in Kiel) were amongst the first, besides Forel and Ule, to use the comparator scale at open sea during the Greenland Expedition of the Geographical Society between 1891-1893 (Die Grönland-Expedition der Gesellschaft für Erdkunde [4]) and the plankton expedition of 1889 (Ergebnisse der in dem Atlantischen Ocean von Mitte Juli bis Anfang November 1889 ausgeführten PlanktonExpedition der Humboldt-Stiftung) [5]. Since then, the scale has become the most commonly used and most simple comparator scale to determine the colour of seas, lakes and rivers.
Numéros de la Gamme Forel.

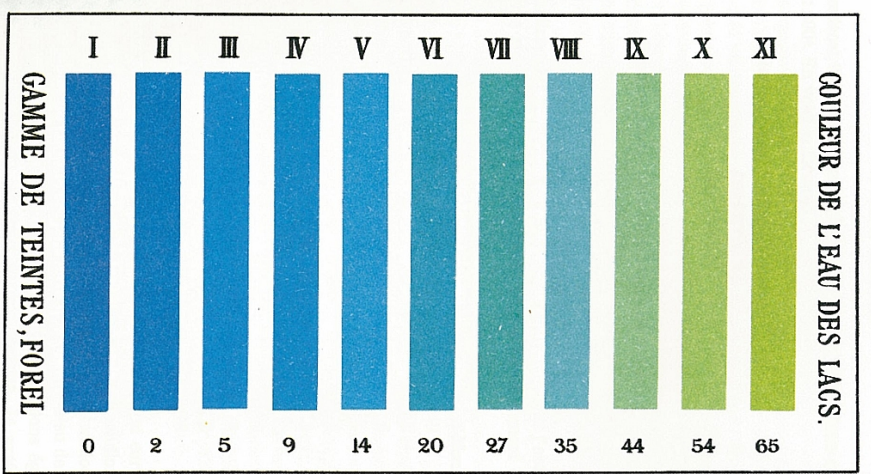

Tant pour cent de solution jaune dans la bleue.

FIG. 1 The tints of the scale as printed in Forel's Volume II of his, three volumes covering, limnology monograph 'Le Léman' published in 1895 [6].

Krümmel spectrally investigated the scale, which was offered to him by Forel, with "a small spectroscope" [7]. From Table 1, it can be seen that the recorded transmission wavelengths are typically $10 \mathrm{~nm}$ apart for the first 6 FU solutions, here expressed as the percentage of Yellow solution added to the basic Blue solution.

The modern version of the FU scale is shown in Figure 3. It comes in two parts, includes an extra tube containing distilled 


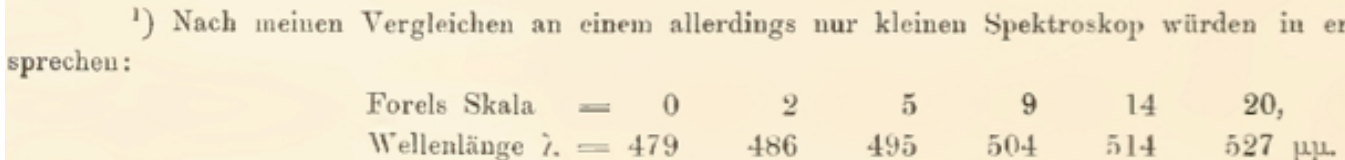

Die Skalenstufe 2 Forels fallt nngefähr mit der Frauenhofer'schen Linie $F$, Stufe 20 mit $E$ zusammen.

TABLE 1 The dominating transmission wavelength for the FU scale numbers 0\% Yellow (FU1) to 20\% Yellow (FU6) as measured by Krümmel in 1893 [7, 8]. At the time, Forel's part of the scale was expressed in percentages of Yellow solution added to the basic Blue solution. Translation: (top) After comparing it with a small spectroscope it matched at first approximation with; (bottom) The scale grade 2 coincides more or less with the Fraunhofer line $\mathrm{F}$, grade 20 with $\mathrm{E}$.
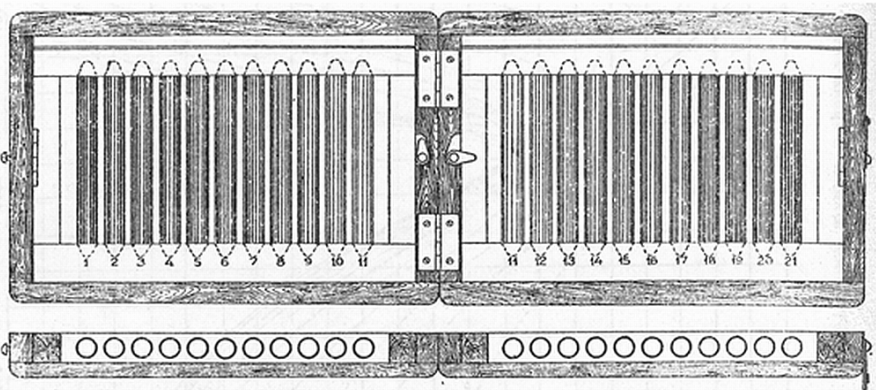

FIG. 2 The FU scale in a protective wooden case, parts can be closed to protect it from sunlight, as proposed by Schokalsky and Nan'niti $[9,10]$.

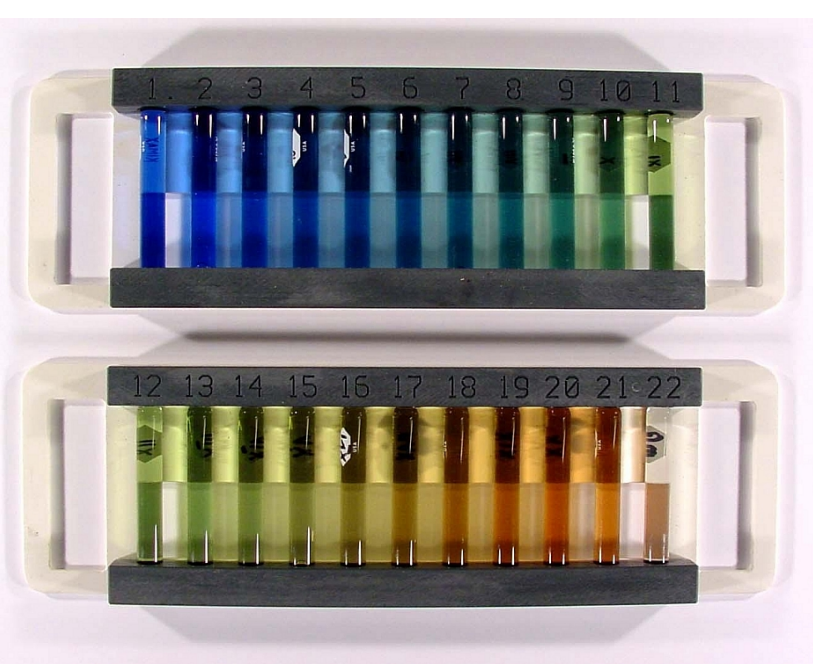

FIG. 3 The modern FU scale with an additional tube filled with distilled water (next to the brown $\mathrm{FU}=21$ ).

water and is kept in the dark, in a refrigerator, to avoid discolouration. In the field, the operation of the comparator scale is almost identical to the procedure suggested by Forel (see Figure 4).

The scale is held above the sea surface and the operator is looking to the water through one of the observation windows next to each tube. The best match between the colour of the water column and one of the tube-colours is established and is documented as an integer number representing the FU scale equivalent. To reduce the effect of reflection of direct sunlight, at the tubes the operator stands in the shadow or under a black umbrella, as Forel himself advised. In order to enhance the signal strength of the water leaving radiance the observation are taken from a submersed Secchi disc [11] (at half the Secchidepth [12]) near the shady side of the vessel. A detailed analysis of the physical and physiological aspects of the Secchi disc can be found in Preisendorfer [13].

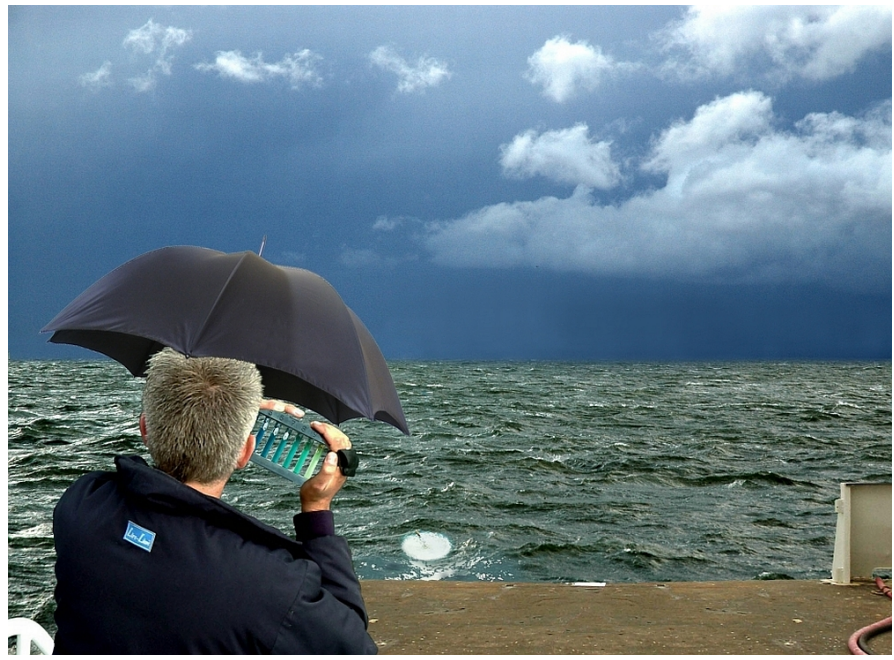

FIG. 4 A FU observation is taken, at the shady side of the vessel, above a submersed Secchi disc, with the disc lowered to a depth of approximately half the Secchi disc depth. The reading of the scale should be done in the shadow.

In this paper, we revisit the spectral properties of the full FU scale. First the reproduction of the scale and the spectral transmission between $380 \mathrm{~nm}$ and $780 \mathrm{~nm}$ (visible part) is described. Subsequently, the transmission curves are transformed to chromaticity coordinates and compared to field samples (see Section 3). The paper concludes with some arguments for continuation of these measurements and intercalibration with modern ocean colour measurements.

\section{METHODS}

\subsection{Forel-Ule recipe and scale dimensions}

The recipe for reproducing the coloured liquids is described in Forel's monograph [14]. A mixture of three standard solutions is used to obtain the colour-palette of the scale. The standard solutions are made with distilled water, ammonia, coppersulphate, potassium-chromate and cobalt-sulphate. The concentrations for the three standard solutions are given in Table 2 and the mixing ratios of the $21 \mathrm{FU}$ scale solutions are presented in Tables 3 and 4. For the Forel part of the scale, FU1 to FU11, mixtures of the solutions Blue and Yellow (see Table 3) are used. The first FU scale colour (FU1) consists for $100 \%$ of the chemical base solution Blue. For the Ule part of the scale, FU12 to FU21, the Green solution, a combination of the mixture $35 \%$ Blue and $65 \%$ Yellow (which gives a green colour) and the Brown solution are mixed to obtain the colour nuances between green and brown (see Table 4). 


\begin{tabular}{|l|l|}
\hline Blue solution: & Amount \\
\hline Copper-sulphate: & 1 gram \\
\hline Ammonia: & $5 \mathrm{ml}$ \\
\hline Distilled water: & $194 \mathrm{ml}$ \\
\hline Yellow solution: & \\
\hline Potassium chromate: & 1 gram \\
\hline Distilled water: & $199 \mathrm{ml}$ \\
\hline Brown solution: & \\
\hline Cobalt-sulphate: & 1 gram \\
\hline Ammonia: & $5 \mathrm{ml}$ \\
\hline Distilled water: & $194 \mathrm{ml}$ \\
\hline
\end{tabular}

TABLE 2 Chemical base solutions of the FU scale.

\begin{tabular}{|c|c|c|}
\hline Solution & \% Blue & \% Yellow \\
\hline 1 & 100 & 0 \\
\hline 2 & 98 & 2 \\
\hline 3 & 95 & 5 \\
\hline 4 & 91 & 9 \\
\hline 5 & 86 & 14 \\
\hline 6 & 80 & 20 \\
\hline 7 & 73 & 27 \\
\hline 8 & 65 & 35 \\
\hline 9 & 56 & 44 \\
\hline 10 & 46 & 54 \\
\hline 11 & 35 & 65 \\
\hline
\end{tabular}

TABLE 3 The mixing proportions (volume \%) of copper-sulphate and potassium chromate to derive the FU scale colours blue to green, respectively FU1 to FU11.

\begin{tabular}{|c|c|c|}
\hline Solution & \% Green & \% Brown \\
\hline 11 & 100 & 0 \\
\hline 12 & 98 & 2 \\
\hline 13 & 95 & 5 \\
\hline 14 & 91 & 9 \\
\hline 15 & 86 & 14 \\
\hline 16 & 80 & 20 \\
\hline 17 & 73 & 27 \\
\hline 18 & 65 & 35 \\
\hline 19 & 56 & 44 \\
\hline 20 & 46 & 54 \\
\hline 21 & 35 & 65 \\
\hline
\end{tabular}

TABLE 4 The mixing proportions (volume \%) of copper-sulphate and potassium chromate to derive the FU scale colours green to brown, respectively FU11 to FU21.

The solutions are filled into glass tubes with a diameter of $10 \mathrm{~mm}$, sealed and fixed in a holder. This holder, shown in Figure 5 , has a dimension of $30 \mathrm{~cm} \times 12 \mathrm{~cm}$, a white background (D, white-opal Perspex or white painted wood) with, half way the tubes, a broad observation window to look through and has a handle on both sides (C). The glass tubes (A) are mounted between two strips (B) with holes.

\subsection{Spectral transmission of the scale}

A TriOS-Ramses spectral radiometer with a FOV of $7^{\circ}$ and a wavelength resolution of $4 \mathrm{~nm}$ was used for the determination of the spectral transmission. With the setup of Figure 6, the ra-

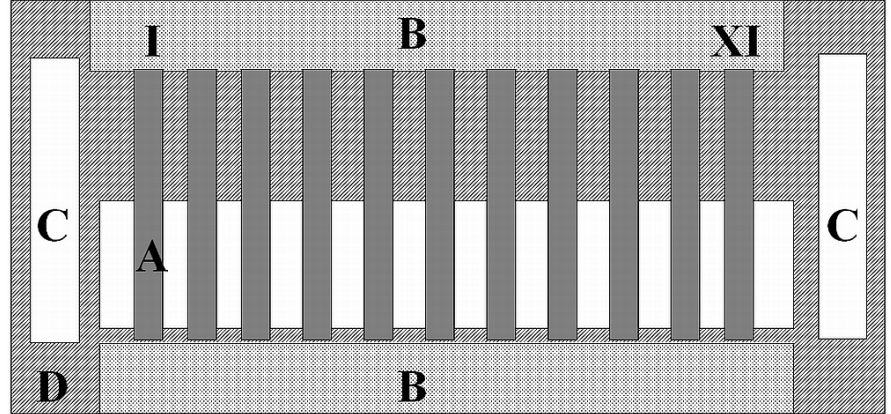

FIG. 5 The layout of half the FU scale. A white Perspex housing (D), with the glass tubes locked between two bars (B). Half the surface behind the tube is closed by white Perspex. The scale can be held by means of the handles at both sides (C). The white rectangle, behind $A$, indicates the open area to look through to the submersed Secchi disc.

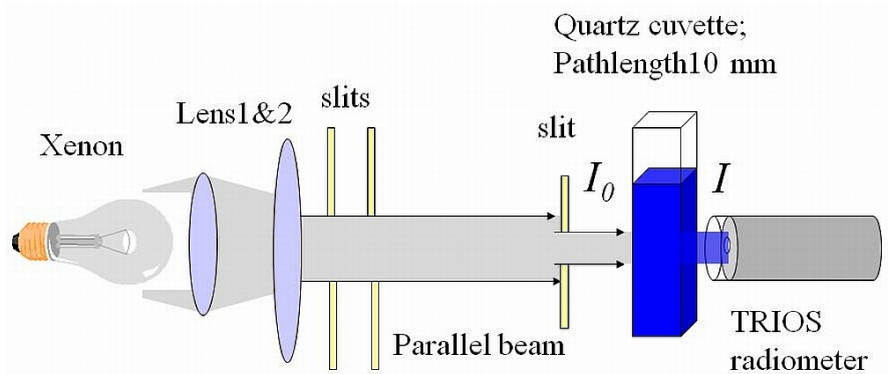

FIG. 6 The spectral transmissions of the Forel-Ule tube colours were determined using a Xenon lamp and a Trios spectral radiometer.

diant flux of wavelength, $\lambda, I(\lambda)$, leaving a cuvette, containing one of the basic solutions or FU mixtures, was determined. $I(\lambda)$ was measured three times for each FU mixture to establish the accuracy of the measurement. During the experiment, the stability of the radiant flux leaving the light source $I_{0}(\lambda)$ was measured before, half way and after the mixture measurements. Per FU mixture, the transmission $T(\lambda)$ was calculated from the ratio of $I(\lambda)$ and $I_{0}(\lambda)$ according to

$$
T_{\mathrm{FU}}(\lambda)=\left(\frac{I(\lambda)}{I_{0}(\lambda)}\right)
$$

For easy comparison, the transmission $T_{\mathrm{FU}}(\lambda)$ was normalised to the maximum of $T_{\mathrm{FU}}$ between $380 \mathrm{~nm}$ and $780 \mathrm{~nm}$ (visible spectrum), where

$$
T_{\mathrm{FU}, \mathrm{MAX}}=\operatorname{MAX}\left(T_{\mathrm{FU}}, \lambda \in[380,780]\right)
$$

and the normalised transmission $T_{\mathrm{FUN}}$ is defined as

$$
T_{\mathrm{FUN}}(\lambda)=\frac{T_{\mathrm{FU}}(\lambda)}{T_{\mathrm{FU}, \mathrm{MAX}}}
$$

\subsection{Colorimetry}

Colorimetry can be described as the science of measuring colour or as "a system for colour measurements". It was introduced in 1931 by the Commission Internationale de l'Eclairage (CIE) or also known in its early days as International Commission on Illumination (ICI). An explanation of colorimetry can be found in Curtis Mobley's book "Light and 
water" [15]. He explains colorimetry as the branch of science, concerned with specifying numerically the colour of a sample of radiant power over the electromagnetic spectrum; i.e. a technique by which an unknown colour is evaluated in terms of standard colours. Apel explains the colour of the sea as the specification of the chromaticity (objective specification of the quality of a colour irrespective of its luminance) of the upward radiance and continues by saying [16]: "it is this intrinsic character that establishes the hue (colour) and the chroma, or strength of the colour of the sea."

Our eye can distinguish colours between dark blue (380 nm) to dark red $(780 \mathrm{~nm})$. A colour can be described as a mixture of three other colours or "tristimuli". The CIE 1931 tristimuli values, called $X, Y$, and $Z$, are parameters derived from the RGB colours [17]. Equal values of $X, Y$, and $Z$ produce white. Tristimuli values can be calculated from the spectral reflectance, transmission or radiance scattered by an object. In this case, seawater or the FU tube colour by the following equations

$$
\begin{aligned}
& X=\int S(\lambda) \bar{x}(\lambda) d \lambda \\
& Y=\int S(\lambda) \bar{y}(\lambda) d \lambda \\
& Z=\int S(\lambda) \bar{z}(\lambda) d \lambda
\end{aligned}
$$

where $S(\lambda)$ stands for the spectral properties of any coloured light source, for instance spectral reflection or spectral transmission, and $\bar{x}, \bar{y}$ and $\bar{z}$ are the 1931 Colour Matching Functions (CMF's). The chromaticity coordinates $x, y$, and $z$ can be calculated by an operation that normalizes out intensity from the ratio of each of the tristimulus values and the sum of the values according to

$$
\begin{aligned}
& x=\frac{X}{X+Y+Z} \\
& y=\frac{Y}{X+Y+Z} \\
& z=\frac{Z}{X+Y+Z}
\end{aligned}
$$

As $x+y+z=1$, and therefore $z=1-x-y$, the third coordinate offers no additional information and only two coordinates (by convention $x$ and $y$ ) are used to represent the colour in a so-called chromaticity diagram as shown in Figure 7 . The outer curved boundary is called the spectral or monochromatic locus with the wavelength in nanometres. The so-called white point $\mathrm{W}$ has the chromaticity coordinates $x=y=z=$ $1 / 3$. If a line is drawn from the white point through a particular $(x, y)$ chromaticity coordinate $\mathrm{F}$, then the ratio of the distance between this point to the white point, distance $(a)$ and the distance from the locus to the white point $(a+b)$ gives us the colour saturation or colour "purity" $(a /(a+b))$ As an example, imagine chalky water and clear water both with the same chlorophyll content. The colour of the water is determined by the amount of chlorophyll present in the water. In case of the chalky water, where the impact of the white calcite on its colour is zero, the $(x, y)$ chromaticity coordinate will be closer to the white point than in the case of clear water. Therefore the saturation of the colour will be less in case of chalky water but the actual colour of the water stays the same.

Due to a possible underestimation of the sensitivity for wave-

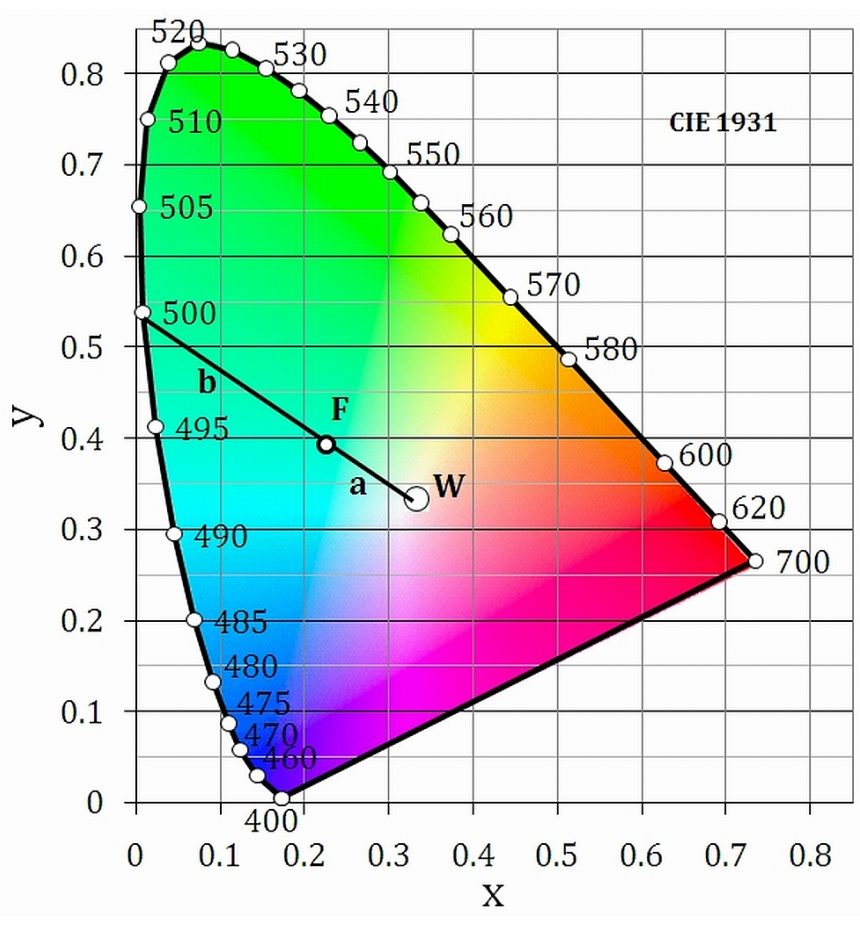

FIG. 7 The CIE1931 chromaticity diagram for monochromatic colours. The outer curved boundary is the spectral or monochromatic locus, with wavelengths in $\mathrm{nm}$. The colour saturation is defined as $a /(a+b)$. $F$ is a specific colour defined by its chromaticity coordinates $(x, y)$.

lengths below $460 \mathrm{~nm}$, Judd [18] derived a new set of CMF's in 1951 which were corrected again by Vos in 1978 [19] and are known as the Judd and Vos-modified CIE 2-degrees colour matching functions. However, an evaluation of the CIE CMFs by Shaw and Fairchild in 2002 [20], resulted in a firm confirmation of the original standard: "Since 1931 the standard has withstood an onslaught of technical pressures and remained a useful international standard."

\subsection{Field measurements}

During several ship cruises on numerous occasions the remote sensing reflectance $R_{\mathrm{RS}}(\lambda)$ [21] was determined by means of a set of TriOS spectrometers [22] with identical spectral response as the laboratory instrument. $R_{\mathrm{RS}}(\lambda)$ is calculate from the ratio of the water leaving radiance $L_{\mathrm{w}}(\lambda)$ and the surface incident irradiance or solar radiation $E s(\lambda)$ according to

$$
R_{\mathrm{RS}}(\lambda)=\frac{L_{\mathrm{W}}(\lambda)}{E S(\lambda)}
$$

Subsequently the $R_{\mathrm{RS}}$ was included as source term in Eq. (4) and the chromaticity coordinates of some different coloured natural water were calculated according to Eq. (5) and presented.

\section{RESULTS}

The spectral transmission of the FU scale has been established from the freshly prepared mixtures as described in Section 2. With the setup shown in Figure 6 the spectral transmission $T_{\mathrm{FUN}}(\lambda)$ for each of the four base solutions (see Figure 8) and for each of the $21 \mathrm{FU}$ solutions was determined (see Figure 9). 


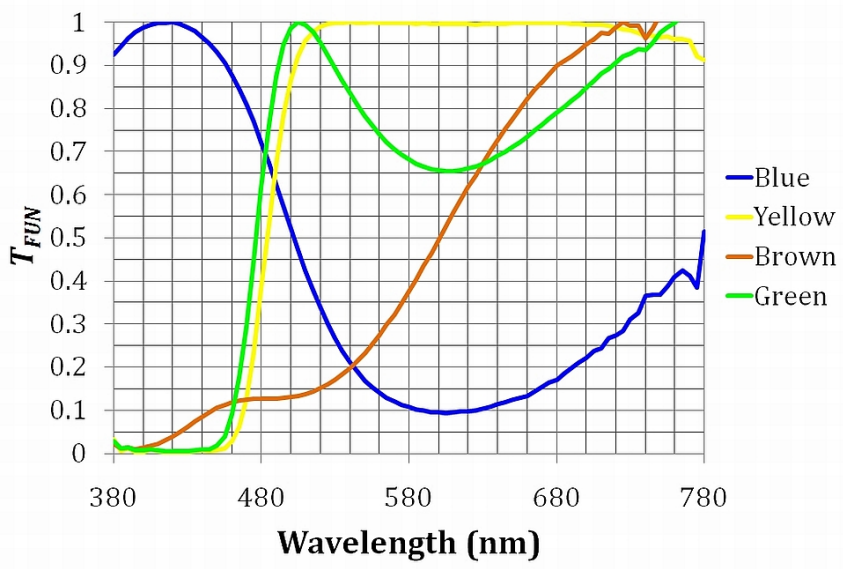

FIG. 8 The spectral transmission of the base solutions Blue, Yellow, Green and Brown of the FU scale as established with a TriOS spectral radio-meter according to the setup of Figure 6 . The spectra were normalised to the maximum transmission between $380 \mathrm{~nm}$ and $780 \mathrm{~nm}$.
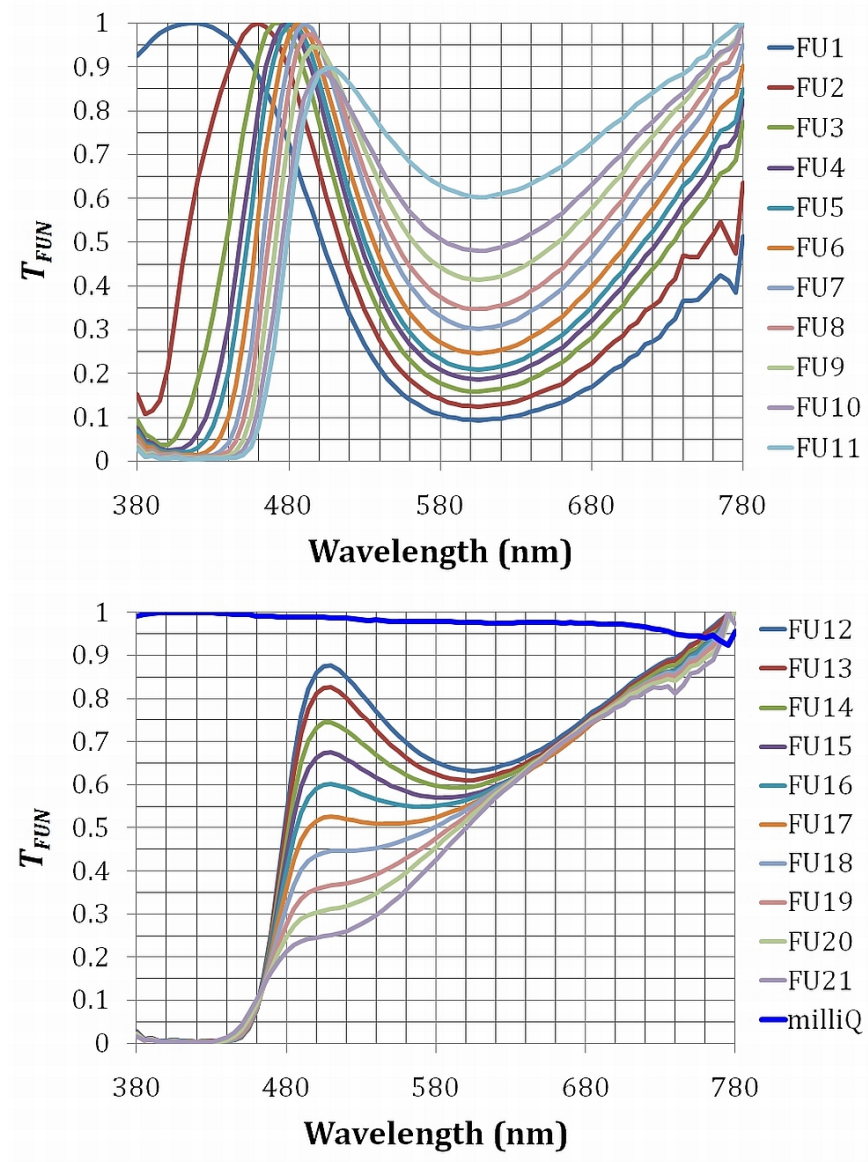

FIG. 9 The normalised transmission $\left(T_{\mathrm{FUN}}\right)$ of the $21 \mathrm{FU}$ tubes plus an extra tube containing purified water (milliQ).

After adding $2 \%$ basic Yellow potassium chromate solution to the basic Blue copper-sulphate (FU1) solution to create FU2, we clearly see a shift in the maximum of the $T_{\mathrm{FUN}}(\lambda)$, from $420 \mathrm{~nm}$ to $465 \mathrm{~nm}$. Figure 9 shows a smooth spectral shift of the peak wavelength, from the blue to the green (FU1 to FU11) in combination with an increase of the transmission above $600 \mathrm{~nm}$. The spectral discrimination between the FU scale colours is clearly visible.
The result of adding the Brown solution to the Green solution for FU12 to FU21, shows a clear decrease in the $T_{F U N}$ at $510 \mathrm{~nm}$. Note that maximum transmission is outside the visual range, resulting in a $\mathrm{T}_{F U N}$ of 1 at $780 \mathrm{~nm}$. The spectrum shifts from green towards the red (brown). In summary the colour shift from FU1 to FU21 can be described as follows; the blue maximum diminishes and gets more pronounced in the green and subsequently the green diminishes and the spectrum of the Brown solutions slowly appears, resulting in a smooth transition from a Blue to a cola Brown solution.

\begin{tabular}{|c|c|c|c|c|c|}
\hline$T_{\text {FUN }}(\lambda)$ & $\mathbf{x}$ & $\mathbf{y}$ & $T_{\text {FUN }}(\lambda)$ & $\mathbf{x}$ & $\mathbf{y}$ \\
\hline FU1 & 0.189 & 0.161 & FU14 & 0.404 & 0.482 \\
\hline FU2 & 0.196 & 0.194 & FU15 & 0.410 & 0.478 \\
\hline FU3 & 0.213 & 0.255 & FU16 & 0.418 & 0.472 \\
\hline FU4 & 0.229 & 0.301 & FU17 & 0.427 & 0.466 \\
\hline FU5 & 0.242 & 0.331 & FU18 & 0.440 & 0.458 \\
\hline FU6 & 0.263 & 0.373 & FU19 & 0.453 & 0.448 \\
\hline FU7 & 0.290 & 0.415 & FU20 & 0.462 & 0.440 \\
\hline FU8 & 0.311 & 0.439 & FU21 & 0.473 & 0.429 \\
\hline FU9 & 0.337 & 0.463 & White & 0.333 & 0.333 \\
\hline FU10 & 0.363 & 0.480 & Blue & 0.189 & 0.161 \\
\hline FU11 & 0.388 & 0.490 & Yellow & 0.436 & 0.496 \\
\hline FU12 & 0.394 & 0.488 & Brown & 0.498 & 0.383 \\
\hline FU13 & 0.397 & 0.486 & Green & 0.386 & 0.489 \\
\hline
\end{tabular}

TABLE 5 The chromaticity coordinates, based on transmission measurements, of the FU scale and the basic solutions. The white refers to the coordinates equal to $1 / 3$.

These colours cover a large range of water types that are found in nature; oligotrophic waters appear indigo-blue and cover the FU scale numbers 1 to 4 , mainly due to the scattering and absorption of pure water. The colour of natural waters changes when more substances are present in the water, like algae, suspended (inorganic and organic) material and dissolved organic material. The colour range of mesotrophic water is approximately bluish green to greenish blue (FU5 to FU9), of eutrophic water greenish blue to yellowish green (FU10 to FU14) and hypereutrophic waters from yellowish green to greenish brown (FU15 to FU18). The last scale numbers (FU19 to FU21) brownish green to brown cover the colour of humic acid dominated waters.

As an example, we compared the chromaticity coordinates of the FU scale with chromaticity coordinates of five natural waters. With a set of TriOS spectrometers we performed at various cruises radiometric measurements for the determination of the remote sensing reflectance $R_{\mathrm{RS}}(\lambda)$. Subsequently the $R_{\mathrm{RS}}$ was calculated according to Eq. (6) and was included as source term in Eq. (4). The chromaticity coordinates of five selected water types were calculated according to Eq. (5), presented in Table 6) and included in Figure 10. The coloured triangles represent the chromaticity coordinates of (from blue to brown) Atlantic Ocean, open North Sea, coastal North Sea, Wadden Sea and a puddle in the woods of the island Texel. By drawing a line from the white point through one of the triangles, representing the chromaticity coordinates of the different water types, we can derive the corresponding FU number. The colour of the Atlantic Ocean can be compared with FU1, the colour of the open North Sea is close to FU3, the coastal North 


\begin{tabular}{|c|c|c|}
\hline $\boldsymbol{R}_{R S}(\lambda)$ & $\mathbf{x}$ & $\mathbf{y}$ \\
\hline Atlantic & 0.170 & 0.150 \\
\hline North Sea - open & 0.210 & 0.230 \\
\hline North Sea -coastal & 0.305 & 0.400 \\
\hline Wadden Sea & 0.380 & 0.430 \\
\hline Puddle & 0.499 & 0.450 \\
\hline
\end{tabular}

TABLE 6 The chromaticity coordinates, based on transmission measurements, of the FU scale and the basic solutions. The white refers to the coordinates equal to $1 / 3$.

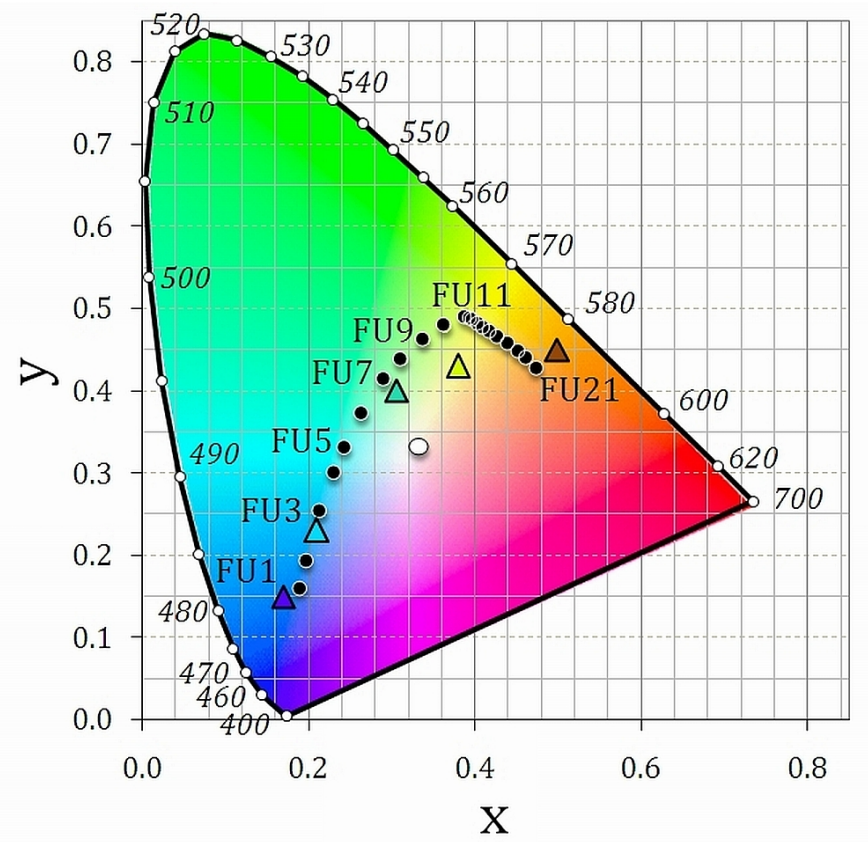

FIG. 10 The CIE1931 chromaticity coordinates, based upon transmission measurements, of the FU scale colours 1 to 21 (black circles) including the white point $(x=y=1 / 3$, white circle). Coloured triangles represent the chromaticity coordinates of, from blue to brown, Atlantic, open North Sea, coastal North Sea, Wadden Sea and a puddle in the woods of the island Texel.

Sea FU7 and the Wadden Sea around FU15. The colour of a puddle in the woods of Texel can be classified as FU21.

Measurements with the FU scale have been part of a yearly returning oceanography course organised at the Royal Netherlands Institute for Sea Research. The reproducibility of the scale was tested by reading the scale at least by 5 different persons over the Wadden Sea. For this shallow tidal basin that receives water from the North Sea and various rivers, FU colour varies between 13 and 17 . The variation in the observation, in one of the five cases, was at most half a scale number. A similar experiment, during an expedition on the Atlantic Ocean (FU varying from 1 to 4 ), again testing the reproducibility of the scale amongst crew members, generated the same result.

\section{CONCLUSIONS AND RECOMMENDATIONS}

The introduction, in the late 1970s, of satellite based radiometers like the Coastal Zone Colour Scanner (CZCS) in 1978 and the introduction of low cost spectral radiometers in the 1980s have shifted the focus of ocean colour measurements away from the FU scale. However, this scale, introduced in the late 19th century as a colour comparator to classify the colour of natural water, is the basis of one of the oldest oceanographic datasets that roughly covers the period 1890-2000. Hundreds of thousands of observations by the FU scale have been collected globally, digitized and stored in the U.S. National Oceanographic Data Centre's World Ocean Database [23].

In this article we provide a reappraisal of the FU scale by analysing the spectral transmission curves and corresponding chromaticity coordinates. Based on this analysis, it can be concluded that the FU scale and its operational use make it a good colour comparator for assessment of the colour of natural waters. The scale has enough spectral discrimination and coverage to classify most of the planet's natural waters. The reconstruction of the scale is simple and is well characterized. In our (limited) tests we have found no marked dependence of the classification on the observer. Of course it remains to be seen if the limited number of scales (21) provides sufficient precision to capture all phenomena in natural waters. Nevertheless, the simplicity, elegancy and robustness to express the colour of water in one number create a simple possibility to compare the past and present status of oceans and inland waters.

We recommend a reintroduction of the FU scale to expand the historical database and to facilitate a tie-in with present satellite ocean colour observations. One option is to mount a Secchi disc on an instrumental- or CTD frame. Thus, for each station both a FU reading and a Secchi depth can be measured and archived together with the already collected historic data.

A link between historic FU observations and satellite observation can be achieved by tranforming MERIS (enough spectral bands) normalized multi-band reflectance image into a FU indexed image.

\section{ACKNOWLEDGEMENTS}

The authors wish to thank Herman Boekel for the remake of the scale, Menno Regeling for his help during preparation and spectral analyses of the scale and Margriet Hiehle for her help during field expeditions and for her inspiring discussions.

\section{References}

[1] G. Field, Chromatography, or, A treatise on colours and pigments, and of their powers in painting, 222 (Tilt and Bogue, London, 1835).

[2] F. A. Forel, "Une nouvelle forme de la gamme de couleur pour l'étude de l'eau des lacs" in Archives des Sciences Physiques et Naturelles/Société de Physique et d'Histoire Naturelle de Cenève, 6, 25 (1890).

[3] W. Ule, "Die bestimmung der Wasserfarbe in den Seen" in Kleinere Mittheilungen. Dr. A. Petermanns Mittheilungen aus Justus Perthes geographischer Anstalt, 70-71 (Justus Perthes, Gotha, 1892).

[4] E. D. von Drygalsi, “Grönland-expedition der Gesellschaft für Erdkunde zu Berlin, 1891-1893 unter leitung von Erich von Drygalsky" in Zweiter Band mit 31 Abbildungen im Text, 10, 318-320, (W. H. Kühl, Berlin, 1897). 
[5] 0. Krümmel, "Die Farbe der Meere" in Geophysikalische Beobachtungen der Plankton-Expedition, Ergebnisse der PlanktonExpedition der Humboldt-Stiftung, 11, 89-110 (Verlag von Lipsius ¿t Tischer, Leipzig, 1893).

[6] F. A. Forel, "Couleur de L'eau" in Optique, Le Léman. Monographie Limnologique, 2, 462-487 (Slatkins, Genève, 1895).

[7] 0. Krümmel, "Die Farbe der Meere" in Geophysikalische Beobachtungen der Plankton-Expedition, Ergebnisse der PlanktonExpedition der Humboldt-Stiftung, 92 (Verlag von Lipsius a Tischer, Leipzig, 1893)

[8] M. Th. Rosen, "Die Skala von Forel-Ule zur Bestimmung der Farbe des Wassers" in Nachrichten des Zentralbüro für Hydrometeorologie, 9, 16-24 (Leningrad, 1930).

[9] M. Th. Rosen, “Die Skala von Forel-Ule zur Bestimmung der Farbe des Wassers" in Nachrichten des Zentralbüro für Hydrometeorologie, 9, 20 (Leningrad, 1930).

[10] T. Nan'niti, "An improvement on the case of Forel's sea color scale" in Meteorological Research Institute, Japan Meteorological Agency, 1, 96 (1953).

[11] P. A. Secchi, "Relazione delle esperienze fatte a bordo della pontificia pirocorvetta Imacolata Concezione per determinare la trasparenza del mare" in Memoria del P.A. Secchi. II Nuovo Cimento Giornale de Fisica, Chimica e Storia Naturale, Ottobre 1864, Published 1865, 205-237 (G.B. Paravia, Torino, 1864).

[12] W. Klinkhardt, Internationale Revue der gesamten Hydrobiologie und Hydrographie, 138 (1943).

[13] R. W. Preisendorfer, "Secchi disk science: Visual optics of natural waters" Limnol. Oceanogr. 31, 909-926 (1986).

[14] F. A. Forel, Le Léman. Monographie Limnologique 2, 464-471 (Slatkins, Genève, 1895).
[15] C. D. Mobley, "Light and water: Radiative transfer in natural waters" 592 (Academic Press, London, 1994).

[16] J. R. Apel, "Principles of ocean optics" in International geophysics series, W. Donn, ed., 38, 586 (Academic Press, London, 1987).

[17] G. G. Wyszecki, and W. S. Stilles, Color science; concepts and methods, quantitative data and formulae, 127-129 (2nd edition, Wiley at Sons, New York, 2000).

[18] D. B. Judd, "Colorimetry and artificial daylight" in Proceedings of the Twelfth Session of the CIE, 1, 11 (Stockholm, 1951).

[19] J. J. Vos, "Colorimetric and Photometric Properties of a $2^{\circ}$ Fundamental Observer" Color Res. Appl. 3, 125-128 (1978).

[20] M. Q. Shaw, and M. D. Fairchild, "Evaluating the CIE 1931 Color Matching Functions" Color Res. Appl. 27, 316-329 (2002).

[21] J. L. Mueller, C. Davis, R. Arnone, R. Frouin, K. Carder, Z. P. Lee, R. G. Steward, S. Hooker, C. D. Mobley, and S. McLean, "AboveWater Radiance and Remote Sensing Reflectance Measurement and Analysis Protocols" in Radiometric Measurements and Data Analysis, Ocean Optics Protocols For Satellite Ocean Color Sensor, Validation, Revision 4, Protocols NASA/TM-2003- 1621/Rev, J. L. Mueller, G. S. Fargion, and C. R. McClain, eds., 3, 21-30 (Greenbelt, 2003).

[22] R. Heuermann, R. Reuter, and R. Willkomm, "RAMSES, A modular multispectral radiometer for light measurements in the UV and VIS" in SPIE proceedings series: Environmental sensing and applications, 3821, 279-285 (Munich, 1999).

[23] T. P. Boyer, J. I. Antonov, H. E. Garcia, D. R. Johnson, R. A. Locarnini, A. V. Mishonov, M. T. Pitcher, 0. K. Baranova, and I. V. Smolyar, "World Ocean Database 2005" in NOAA National Oceanographic Data Center (U.S. Government Printing Office, Washington, 2006). 